\title{
Errata: Dynamic displacement measurements with a stabilized fiber Michelson interferometer based on quadrature-phase-tracking technique
}

\author{
Zhimin Chen \\ Fang Xie \\ Min Li \\ Qibo Feng \\ Beijing Jiaotong University \\ School of Science \\ Department of Physics \\ Optical Science and Technology Laboratory \\ Beijing 100044 China \\ E-mail: fxie@ bjtu.edu.cn
}

This article [Opt. Eng. 49, 013601 (2010)] was originally published on 7 January 2010 with an error in the title. The word "quadrature" appeared incorrectly as "quadratrue."

All online versions of the article were corrected on 16 June 2010. 